\title{
A Petri net model for railway bridge maintenance
}

\begin{tabular}{|c|c|}
\hline Journal: & Part O: Journal of Risk and Reliability \\
\hline Manuscript ID & Draft \\
\hline Manuscript Type: & Original Article \\
\hline Date Submitted by the Author: & $\mathrm{n} / \mathrm{a}$ \\
\hline Complete List of Authors: & $\begin{array}{l}\text { Le, Bryant Linh; University of Nottingham, Civil Engineering } \\
\text { Andrews, John; University of Nottingham, } \\
\text { Fecarotti, Claudia; University of Nottingham, Civil Engineering }\end{array}$ \\
\hline Keywords: & $\begin{array}{l}\text { bridge, Asset management, degradation, lifetime analysis, Weibull } \\
\text { distribution, Petri net, Monte Carlo simulation }\end{array}$ \\
\hline Abstract: & $\begin{array}{l}\text { This paper describes the application of the Petri Net modelling approach to } \\
\text { managing the maintenance process of railway bridges. The Petri Net model } \\
\text { accounts for the degradation, inspection and repair processes of individual } \\
\text { bridge elements in investigating the effectiveness of alternative } \\
\text { maintenance strategies. The times governing the degradation and repair } \\
\text { processes considered are stochastic and defined by the appropriate Weibull } \\
\text { distribution. The model offers a capability for modelling the bridge asset } \\
\text { which overcomes the limitations in the currently used modelling techniques } \\
\text { reported in the literature. The bridge model also provides a means of } \\
\text { predicting the future asset condition as a result of adopting different } \\
\text { maintenance strategies. The solution of the Petri Net model is performed } \\
\text { using a Monte Carlo simulation routine. The application of the model to a } \\
\text { typical metal railway bridge is also presented in the paper. }\end{array}$ \\
\hline
\end{tabular}

\section{SCHOLARONE"}

Manuscripts 


\title{
A Petri Net Model for Railway Bridge Maintenance
}

\author{
Bryant Le, John Andrews and Claudia Fecarotti \\ Infrastructure Asset Management Group, University of Nottingham
}

\begin{abstract}
This paper describes the application of the Petri Net modelling approach to managing the maintenance process of railway bridges. The Petri Net model accounts for the degradation, inspection and repair processes of individual bridge elements in investigating the effectiveness of alternative maintenance strategies. The times governing the degradation and repair processes considered are stochastic and defined by the appropriate Weibull distribution. The model offers a capability for modelling the bridge asset which overcomes the limitations in the currently used modelling techniques reported in the literature. The bridge model also provides a means of predicting the future asset condition as a result of adopting different maintenance strategies. The solution of the Petri Net model is performed using a Monte Carlo simulation routine. The application of the model to a typical metal railway bridge is also presented in the paper.
\end{abstract}

Keywords: bridge, asset management, degradation, lifetime analysis, Weibull distribution, Petri net, Monte Carlo. 


\section{Introduction}

The UK railway network operates and maintains more than 35,000 bridges. A large proportion of this bridge population was constructed more than 100 years ago and many of them were not originally designed to meet the current network demand experienced. The higher traffic frequency, speed and loads occurring on the network are expected to result in an increasing rate of deterioration for the structures. This provides a significant challenge when considered alongside the desire to minimise expenditure and traffic disruption resulting from activities to manage the state of these assets. As a consequence a greater emphasis has been given to the strategies for asset maintenance.

Although the modelling approach used in this paper is generic, the focus is on the asset group of metal underbridges which make up $45 \%$ of the UK railway bridge population. An underbridge is a type of bridge that carries traffic over obstacles. Metal structures deteriorate faster than those of concrete or masonry construction making metal underbridges one of the most critical asset groups on the railway. An accurate model is required to predict the future asset condition resulting from different maintenance strategies.

The first bridge condition models were developed over 30 years ago and several models now exist which provide support tools for bridge asset management. These models can be considered in three difference categories: Markov, semi-Markov and lifetime analysis based models. All of the models predict the performance for the entire bridge or the bridge components. In both the Markov and semi-Markov models, the deterioration rate of the bridge or bridge element is reflected in the transition probabilities [1]. Jiang \& Sinha [2] and Robelin \& Madanat [3] explained the use of Markov models in predicting the deterioration rate of bridges. Cesare et al. [4], Ortiz-Garcia et al. [5] and Chase \& Gáspár [6] present applications of Markov models to the evaluation of bridge deterioration. Fernando et al. [7] and Lethanh et al. [8] utilise Markov approach to evaluate and optimise the intervention strategies on bridges. While the Markov model is based on the assumption of an exponential distribution for the duration (sojourn) times in specific bridge conditions, semi-Markov models use different distributions (often the Weibull distribution) to model these duration times. Ng \& Moses [9] discussed the use of semi-Markov processes in modelling bridge deterioration. Yang et al. [10] presented and tested a framework model predicting bridge condition using the semi-Markov approach. It suggests that the model is more suitable than the traditional Markov model. Lifetime analysis based models were developed by Agrawal et al. [11], where over 17,000 highway bridges in New York State were studied with historical data available from 1981 to 2008. The approach fits a Weibull distribution to the durations that a bridge element stays in a particular condition and then calculates the mean time to reside of that state. The mean duration for each different condition rating is calculated by accumulating the mean durations of the previous states. These means are then plotted on a graph of condition ratings against age and a third degree polynomial fitted to show the deterioration rate.

In the literature, Markov models have proved to be the most popular structural modelling approach and this has been used for more than 20 years to predict the degradation of bridges. 
However, the fundamental Markov property only allows bridges or bridge components to experience a constant deterioration rate. Semi-Markov models overcome this limitation, however, due to their increased complexity they have only been applied to simple problems. The semi-Markov approach also suffers from a similar limitation of the traditional Markov approach such as the size of the model increases dramatically with the number of model states. Furthermore, for complex problems, the estimation of transition probabilities will require a significant computation time [12]. In lifetime analysis based models, the degradation process of bridges or bridge elements is modelled based on the lifetime analysis technique $[13,14]$. An appropriate distribution is selected to model the times of a bridge component reaching a specified condition state. This approach considers both complete and incomplete lifetime data. It was demonstrated that the Weibull distribution is a good fit to the lifetime data [15], also the Weibull distribution parameters obtained indicate a non-constant i.e. increasing deterioration rates of bridge elements [16]. Many studies have applied the method to model the degradation process between different states however a complete bridge model comprising of individual components and their condition states has not been developed.

The majority of the models in the literature use condition rating scores to determine the degradation processes. This has severe limitations. The condition score is considered subjective [17] and does not provide the necessary detail needed in the degradation information to relate it to the maintenance actions required to rectify the condition. Limitations associated with the use of condition ratings to derive the degradation parameters were also discussed in the literature. Agrawal, Kawaguchi \& Chen [11], note that the estimation of the transition probabilities is significantly affected by maintenance actions carried out between inspections which result in a rise in the condition score. These effects of maintenance on components are not captured. De Stefano \& Grivas [18] also indicate that the actual date at which a state transition event occurs is unknown because inspections are performed only periodically (in many cases they are many years apart). It is often assumed that the transition event occurs at midpoint between the corresponding inspection dates, thus the accuracy of the model is reduced when the inspection intervals are large.

The bridge model developed in this paper addresses the deficiencies outlined above by employing the Petri Net method to model the structure. Petri Nets provide a stochastic technique which allows far greater detail in the modelling of the bridge elements in comparison to the alternatives whilst maintaining a manageable model size. A bridge model is developed taking into account the degradation, inspection, maintenance/renewal processes and the interaction between these processes. For the degradation processes, the limitations associating with condition data is avoided by the use of historical maintenance data. The study of historical maintenance data relating the maintenance actions with certain types of defect conditions and the time period experienced to achieving the condition gives a better understanding of the variability of the deterioration process [16]. Weibull distributions are used to model the distribution of component life times to reaching specified condition states. The model is also capable of accounting for different maintenance strategies, inspection, servicing intervals, repair planning time and maintenance schedule. 


\section{Degradation analysis of bridge elements}

The defect types which occur on the structures are different for each element type and material. Therefore every maintenance action is specific to the requirements of the task to be performed and the bridge element it is to be performed on. Based on the extent of defect being repaired, according to the duration and costs of the work, the intervention actions considered are classified as: minor repair, major repair and replacement. They are carried out when the component reaches the good, poor or very poor state respectively from the 'as $n e w$ ' condition. These interventions are assumed to restore the component condition to 'as good as new'.

The degradation of a bridge element is analysed by studying the historical maintenance records throughout its lifetime. The detail of the lifetime analysis is discussed in [16] and [19]. Having obtained the lifetime data for bridge components, components of the same type and materials have been grouped together and, assuming the data to come from a homogeneous sample, a two-parameter Weibull distribution used to model the time to deteriorate to the specified defect characteristic of that particular element. For the twoparameter Weibull distribution, the expression for the pdf is:

$$
f(t)=\frac{\beta}{\eta}\left(\frac{t}{\eta}\right)^{\beta-1} e^{-\left(\frac{t}{\eta}\right)^{\beta}}
$$

where

$\beta$ is the shape parameter

$\eta$ is the scale parameter

The Weibull distribution parameters obtained for different major bridge components are shown in Table 1. In general, the $\beta$ parameter of the distributions obtained suggests a slight increase in the component deterioration rates over time since it commonly has a value greater than one. For example, the Weibull shape parameter obtained for the main girder illustrates that the rate of deteriorating from the 'as new' to a good condition is increasing with time (wear-out characteristic) and has a mean time in the 'good' state of nearly 21 years. In the case where the data is not available to obtain the distribution (as noted by the $\left(^{*}\right)$ in Table 1 ), the rates were estimated using bridge expert's opinions.

\begin{tabular}{|c|c|c|c|c|c|c|}
\hline \multicolumn{7}{|c|}{ Weibull Fitting (Weibull 2-parameters) } \\
\hline Bridge component & Material & Condition & Intervention & Beta & Eta (year) & Mean (year) \\
\hline \multirow{3}{*}{ GIRDER } & \multirow{3}{*}{ Metal } & Good & Minor Repair & 1.71 & 23.39 & 20.86 \\
\cline { 3 - 7 } & & Poor & Major Repair & 0.87 & 44.27 & 47.49 \\
\cline { 3 - 7 } & & Very Poor* & Replacement* & 1.14 & 149.63 & 142.77 \\
\hline \multirow{3}{*}{ DECK } & \multirow{3}{*}{ Metal } & Good & Minor Repair & 1.265 & 10.28 & 9.54 \\
\cline { 3 - 7 } & & Poor & Major Repair & 1.038 & 20.00 & 19.71 \\
\cline { 3 - 7 } & & Very Poor & Replacement & 1.009 & 28.47 & 28.36 \\
\cline { 3 - 7 } & Concrete & Good & Minor Repair & 1.082 & 19.09 & 18.52 \\
\hline
\end{tabular}




\begin{tabular}{|c|c|c|c|c|c|c|}
\hline \multirow{4}{*}{ BEARING } & Poor* & Major Repair* & 1.000 & 26.67 & 26.67 \\
\cline { 3 - 7 } & Very Poor & Replacement & 0.976 & 34.26 & 34.63 \\
\cline { 3 - 7 } & \multirow{3}{*}{ Timber } & Good & Minor Repair & 1.312 & 3.99 & 3.68 \\
\cline { 3 - 7 } & & Poor & Major Repair & 1.371 & 7.13 & 6.52 \\
\cline { 3 - 7 } & & Very Poor & Replacement & 1.501 & 6.12 & 5.52 \\
\hline \multirow{3}{*}{ ABUTMENT } & Good & Minor Repair & 0.838 & 14.94 & 16.41 \\
\hline & \multirow{3}{*}{ Masonry } & Poor & Major Repair & 2.129 & 14.43 & 12.78 \\
\cline { 3 - 7 } & & Very Poor* & Replacement & 1.000 & 21.92 & 21.92 \\
\hline & & Good & Minor Repair & 1.000 & 51.94 & 51.94 \\
\cline { 3 - 7 } & & Poor* & Major Repair* & 1.000 & 100.87 & 100.87 \\
\cline { 3 - 7 } & Very Poor* & Replacement* & 1.000 & 150.00 & 150.00 \\
\hline
\end{tabular}

Table 1: Distribution results of studied bridge components (* Estimated rates using bridge experts' opinions)

\section{Petri Net definition}

C. A. Petri [20] developed the Petri Net (PN) in 1962 as a tool for representing dynamic processes using directed graphs. The graphs could then be simulated to analyse the performance of the system. This method is gaining in popularity due to its flexibility for modelling dynamic systems and has been used in many fields such as engineering, science and business [21-23]. A Petri Net consists of basic elements: places, transitions, arcs and tokens. A place represents a condition or event in the system and is denoted by a circle on the graph. A token denoted by a dot is located in a place to represent the presence of that condition. A transition allows the token to move between places to model the changing condition of the system and appears as a rectangle on the graph. Places and transitions are linked by arcs (represented by arrows). The direction of the arc indicates the input and output places for a transition. In a Petri Net, the state of the system is characterised by the marking of the tokens in the places. Consequently, the changes in the marking of the net describe the state changes in a system. Changes in the system state are represented by the firing of enabled transitions which remove input place tokens and create output place tokens according to the firing rules. This process enables the Petri Net to model the dynamic behaviour of the system [24]. The Petri Net also allows a proper representation of the dynamic interactions between different system's components which influence the system behaviour and its maintenance [25].
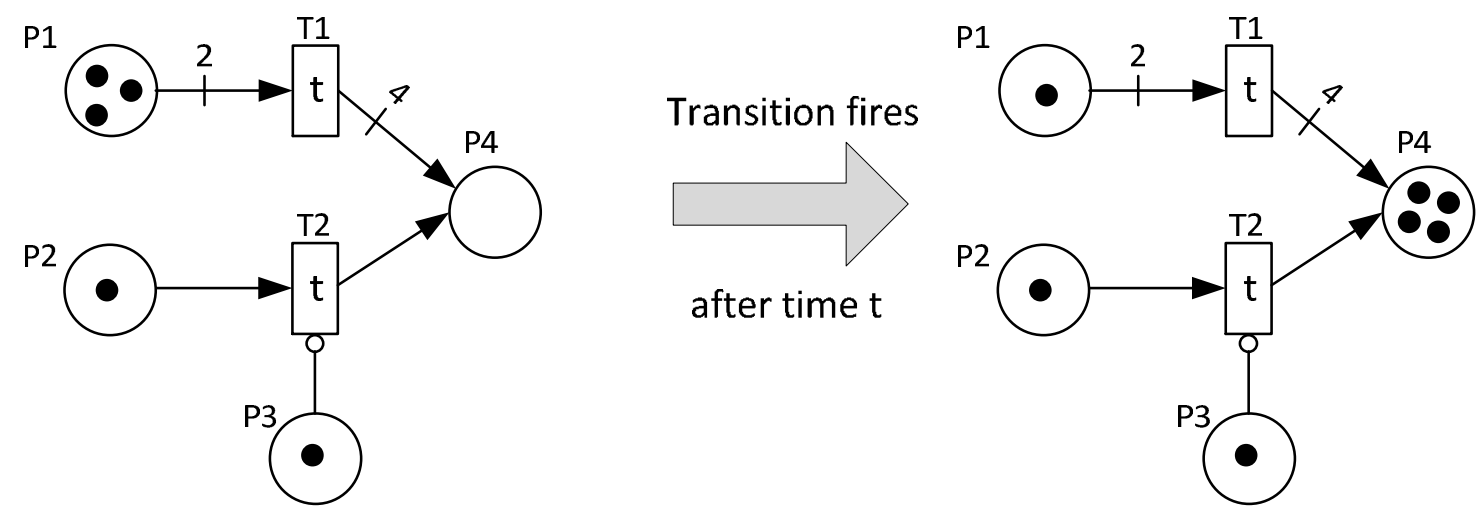
Figure 1: Simple PN with arc multiplication and inhibitor arc and the firing process.

A simple, traditional, PN is illustrated in Figure 1. The positive integer associated to an arc is called the arc multiplicity [26]. If the arc is an input arc from a place to a transition then the arc multiplicity dictates the number of tokens needed for the transition to be enabled. If the arc is an output arc from a transition to a place, the arc multiplicity indicates the number of tokens that will be deposited in the output place. Once the transition is enabled (all input places marked with the appropriate number of tokens) then after the time dictated for the transition, $t$, for $\mathrm{T} 1$, the transition fires and removes the multiplicity of tokens from the input places and deposits the multiplicity of tokens to the outputs. This is indicated by the PN on the right of Figure 1. An inhibitor arc [27] can only go from a place to a transition and is denoted as an arc with a round end. When the input place P3 is marked with a token, the transition T2 is inhibited and will not fire as long as the token in place P3 remains. This is the reason why after time $t$, the token in place $\mathrm{P} 2$ remains as the transition $\mathrm{T} 2$ is inhibited from firing by a token in place P3. The inhibitor arc may also have a multiplicity, in this case, the place must contain at least the number of tokens as indicated by the arc multiplicity for the transition to be inhibited.

The Petri Net used in this paper can be regarded as an extension to the traditional PN. Some features of the net are added to enable an efficient representation of the problem of modelling a bridge maintenance process and they are discussed below.

\section{Token}

For the models developed, the degradation of different elements of the bridge has the same model structure. To avoid the need to duplicate the PN for each element the same structure is used with different tokens to represent each particular bridge element. A token is unique, with an associated ID represented by their different 'colour'. A token also carries a set of properties with it, they are: token ID, component type/name, component material, coating condition, environment factor, and type of repairs required (if the component is in a condition that triggers repair).

\section{Transitions}

Special transitions have been used in order to create the model representation of the bridge. For these new transitions, the enabling and firing processes are the same as those for the traditional PN method, however, transitions also contain properties to implement specific tasks. Although these tasks can be completed using traditional PN, these additional features help to make the model more concise and efficient.

- Conditional transition: All transitions used require this property to accommodate the unique aspect of the tokens. This is to make sure that the dynamic movement of tokens around the PN occurs according to appropriate deterioration and repair distributions and that each token is independent from each other. Figure 2 illustrates this feature with an example net with transition T1 is enabled. After time $a$, the white token is fired and after time $b$, the dark token is fired. These times are sampled from 
different distributions as the tokens represent different items. This is different to conventional PN, as the transition time would usually be sampled from the same distribution. In the bridge model, this property ensures that different bridge elements follow their own deterioration and repair processes.
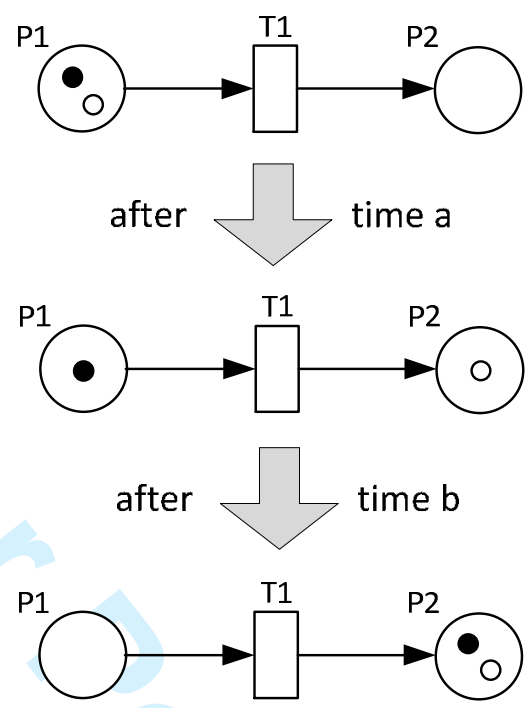

Figure 2: Conditional transition

- Periodic (PD) transition: With this transition firing only occurs when the system time is at a specified value. In the model, this transition is used to represent the inspection process where the condition of the bridge element is revealed after inspection after a specified time interval. It is also used to model the maintenance schedule for an asset so the repair can only happen at these times.

- Reset (RST) transition [28]: When this transition fires, it resets the marking of specified places in the PN to some desired state. This transition has an associated list of places and number of tokens that they will contain after reset. A reset action on a network can be carried out using conventional PN features but would require a large number of transitions and places to be added which would increase the size and complexity of the model and confuse the overall structure.

The symbols used to represent the periodic and reset transitions are given in Figure 3.

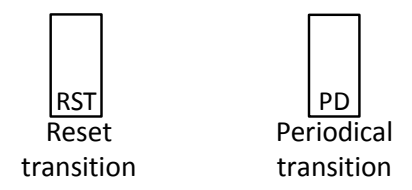

Figure 3: Representation of transitions with reset and periodical property. 


\section{Petri Net bridge model}

\subsection{Degradation process}

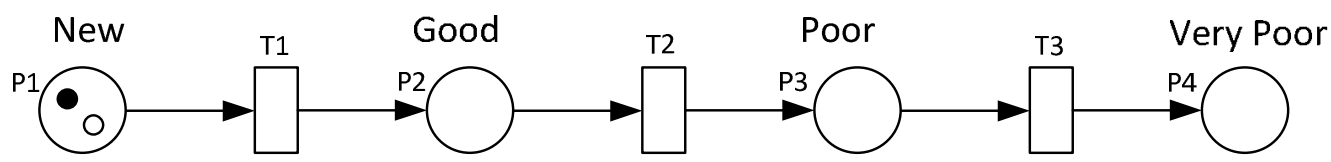

Figure 4: Petri-Net models the degradation process

In the model, bridge components have been considered to reside in one of four conditions which are: as new, good, poor and very poor condition. The component starts in the new condition and degrades to a good condition which could be restored to 'as new' through minor repair. Further deterioration leads to the poor and very poor conditions where the component requires major repair and complete renewal to return it to 'as new' respectively. The degradation process can be represented using the net as illustrated in Figure 4. Places P1 to P4 represent the four condition states and the transitions between these states are represented by conditional transition $\mathrm{T} 1$ to T3. In Figure 4, two different bridge components are represented by two different coloured tokens. The transitions times for the black and white tokens are sampled from the appropriate Weibull deterioration distributions discussed in the previous section. These conditional transitions ensure that different bridge components follow their unique deterioration processes.

\subsection{Modelling dependent deterioration processes}

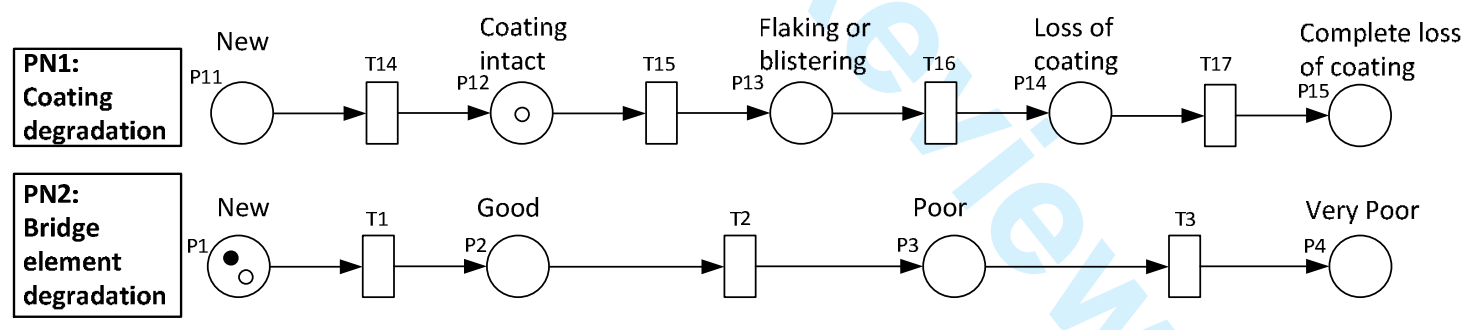

Figure 5: Petri-Net models metal element include the condition of coatings

The PN capability of modelling dependent deterioration processes is demonstrated through the modelling of the degradation of the protective coating and its effect on the deterioration rate of its protected metal. Figure 5 shows two separate PNs, the top net models the condition of the coating and the bottom net models the condition of the metal bridge element that the coating is protecting. There are five coating conditions which are represented by places P11 to P15. The transitions between these conditions are represented by transitions T14 to T17. Even though the two nets are separated however the modelling of the deterioration processes of the element and its coating are dependent. This is modelled by connecting the token in both nets through their characteristics. In the net PN1, the position of the token updates that token property. This property i.e. the condition of the coating at any time is therefore also 
captured in the token in the net PN2. Based on the property information of the token, an appropriate rate is chosen to model the deterioration of the metal element in the net PN2.

\subsection{Inspection process}

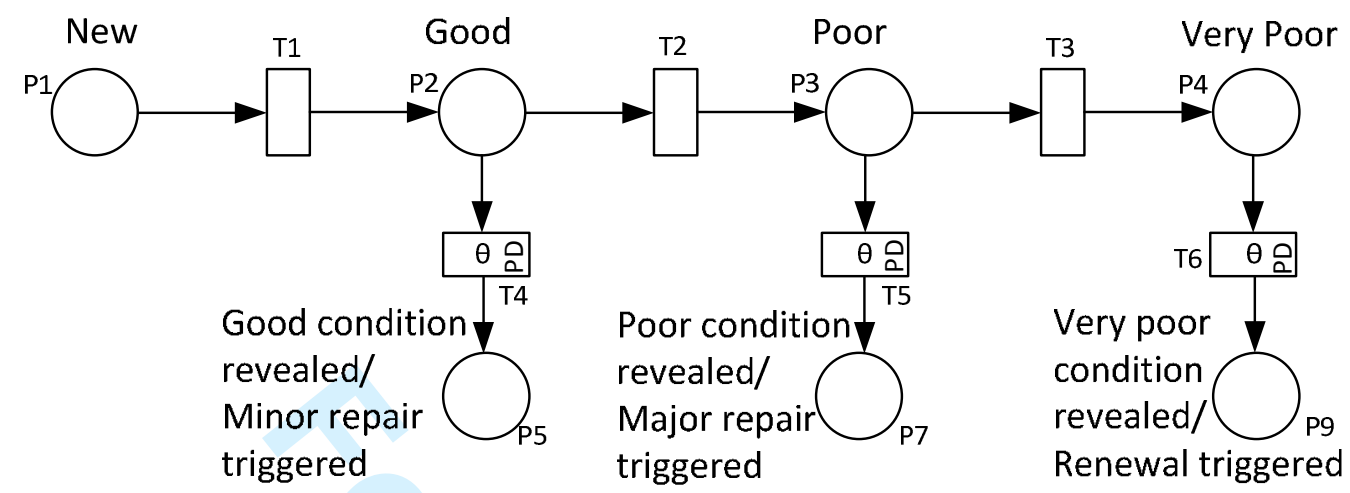

Figure 6: Inspection process

All bridges and their components are inspected after a specified period of time at which point the true condition of the component is revealed. Appropriate maintenance work can be performed or the component allowed to deteriorate further. The inspection process is modelled in the PN by the periodical transitions T4, T5 and T6 as illustrated in Figure 6. The inspection time is set using these transitions. Assuming the inspection time is set at every $\theta$ time units and the component has reached good condition (P2), there are two possible pathways from this state which are represented by transitions T2 and T4. If transition T4 is fired first, the token is transferred to place P5 which means the component is now been inspected and the condition revealed as good. However if transition T2 is fired first, the token is transferred to place P3 which means the component has degraded to the poor condition (P3) before it was inspected. The transition time of transition T4 is the time to inspection i.e. the time between which the token arrives at P2 and the time when the component is next inspected at $\theta, 2 \theta, 3 \theta \ldots n \theta$. Similarly, the periodical transition T5 and T6 are used to determine when the component reaches the poor and very poor states. The inspection process is also applied to the net PN1 in Figure 5 so that the state of the coating is revealed following inspection.

\subsection{Maintenance process}

When the condition of the bridge element is identified, appropriate maintenance action can be triggered to restore the element condition to as good as new. The maintenance process does not usually happen immediately and often has an associated planning time, this repair planning time depends, not only on the bridge management authority, but also (if necessary) on the maintenance schedule of the line as some interventions can only be carried out when there are no trains running. Moreover, in practice, maintenance actions are often carried out according to a schedule that is set by the authority considering the maintenance of different parts on the railway network (e.g. route criticality, the condition of railway line, etc.). Therefore maintenance actions usually follow a planned delay period and follow a specific maintenance schedule which needs to be accounted for in the model. 
The structure of the PN which models the maintenance process for bridge components can be seen in Figure 7. Places P5, P7 and P9 represent states for the true bridge condition which has been revealed following an inspection. When a token is present in any of these places, this means that the corresponding repair is scheduled. Transitions T7, T8 and T9 represent the repair planning time for each type of repair (minor repair, major repair and renewal respectively). Place P10 is effectively the job list that is to be carried out at the next maintenance schedule available which is set by the periodic transition T10. In particular, transition T10 governs the list of times that the transition is allowed to fire, this list of times corresponds to the maintenance schedule for the asset. The maintenance schedule also includes the actual repair time (the time between when the work starts and finishes). The conditional property of the transition $\mathrm{T} 10$ ensures that the appropriate repair times are generated for different bridge components requiring different repairs. Following a repair, transition T10 fires which transfers the token to place P1 implying the condition of the element is restored to the as good as new condition. The net presented is capable of modelling different repair planning times for different components, and any components that are awaiting maintenance in the job list would be repaired at the next available maintenance schedule.

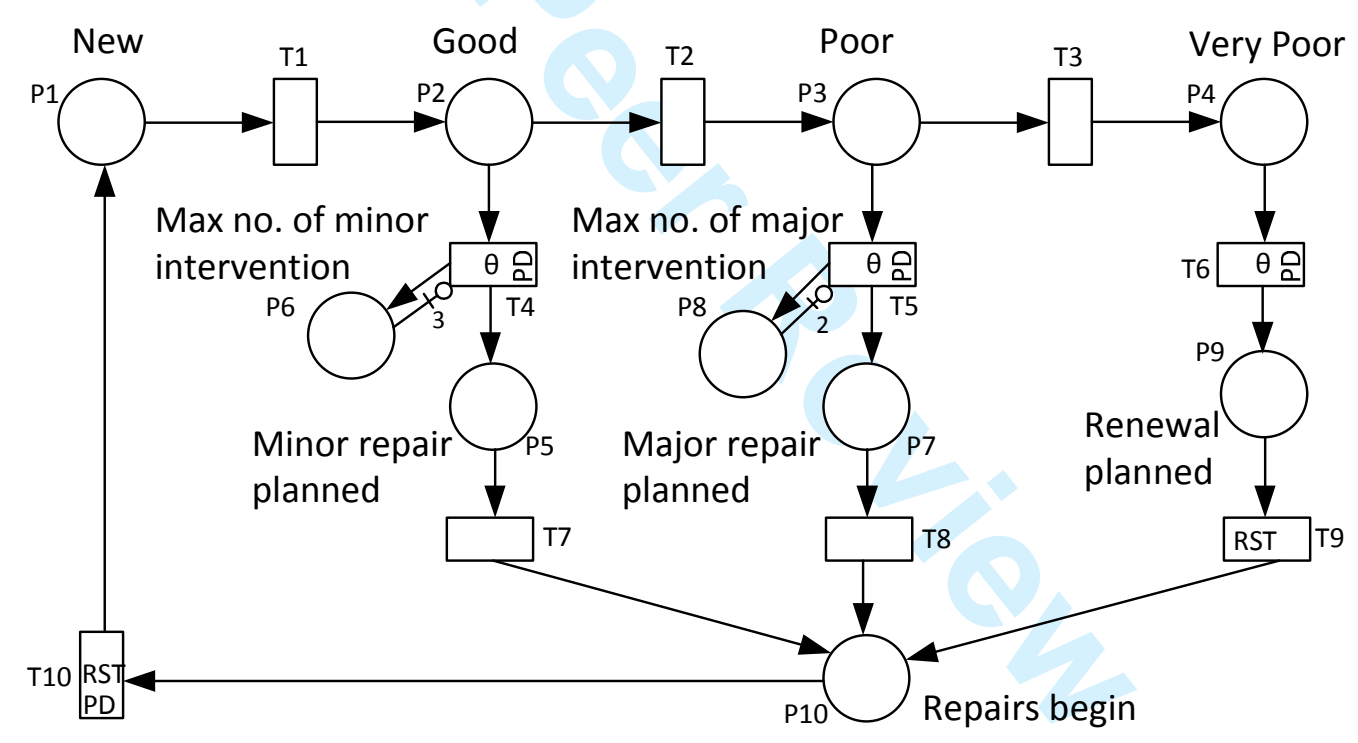

Figure 7: Intervention and repair planning process

The inability to model the effectiveness of maintenance is a common feature in asset management models in the literature, including those for bridges. A common assumption is that repairs always restore the condition of bridge components regardless of the maintenance history. This implies that as long as some form of maintenance is performed, the bridge component will never reach a point where it needs replacement. This assumption is not practical, especially, in whole life cycle costing analysis. The PN method, however, is capable of modelling such situations. In this model it is assumed that minor repair becomes ineffective after three times of being carried out and major repair is only effective for two times. This means that over the component life, once these conditions have been satisfied, the component can only degrade further to the very poor state at which point a replacement is 
necessary. The rules are implemented in the PN by introducing the places (P6 and P8 in Figure 7) which record the number of times a certain type of maintenance has happened and inhibit the repair process once the maximum number of that type of intervention is reached. Assuming the bridge element is in a good condition (P2) when it is inspected, this means the transition T4 fires and the token is now removed from $\mathrm{P} 2$ and marked in place P5 and P6. The number of tokens in place P6 indicates the number of minor repairs that have occurred over the component life time. Place P6 connects with transition T4 by an inhibitor arc with the multiplicity of 3 . Thus when there are three tokens of the same type in place P6, the transition T4 will be inhibited and minor interventions are not possible anymore. Similarly, place P8 records the number of major repairs that has happened.. Note that following a component replacement (place P9), the history of the number of minor and major interventions recorded in places P6 and P8 should be cleared. This is implemented in the model by using the reset property in transition T9.

Transition T10 in Figure 7 is a reset transition, which when it fires, resets the net by removing all tokens in any place and marks one token in place P11 (Figure 8). This reset action in the model implies that when the repair happens on the metal element, the coating of that element is also restored to a new condition. The restoration of the coating also follows another independent repainting process (shown in Figure 8) that is triggered after a predetermined durations. Transitions T18 to T21 are periodical transitions used to model repainting every $\phi$ years.

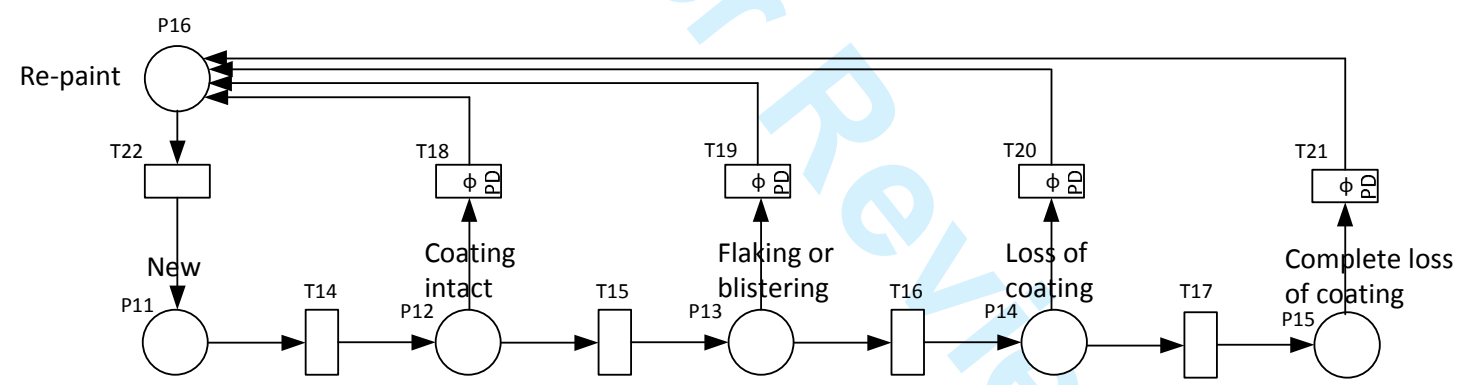

Figure 8: Repainting process for coating of metal element. 


\subsection{Opportunistic maintenance}

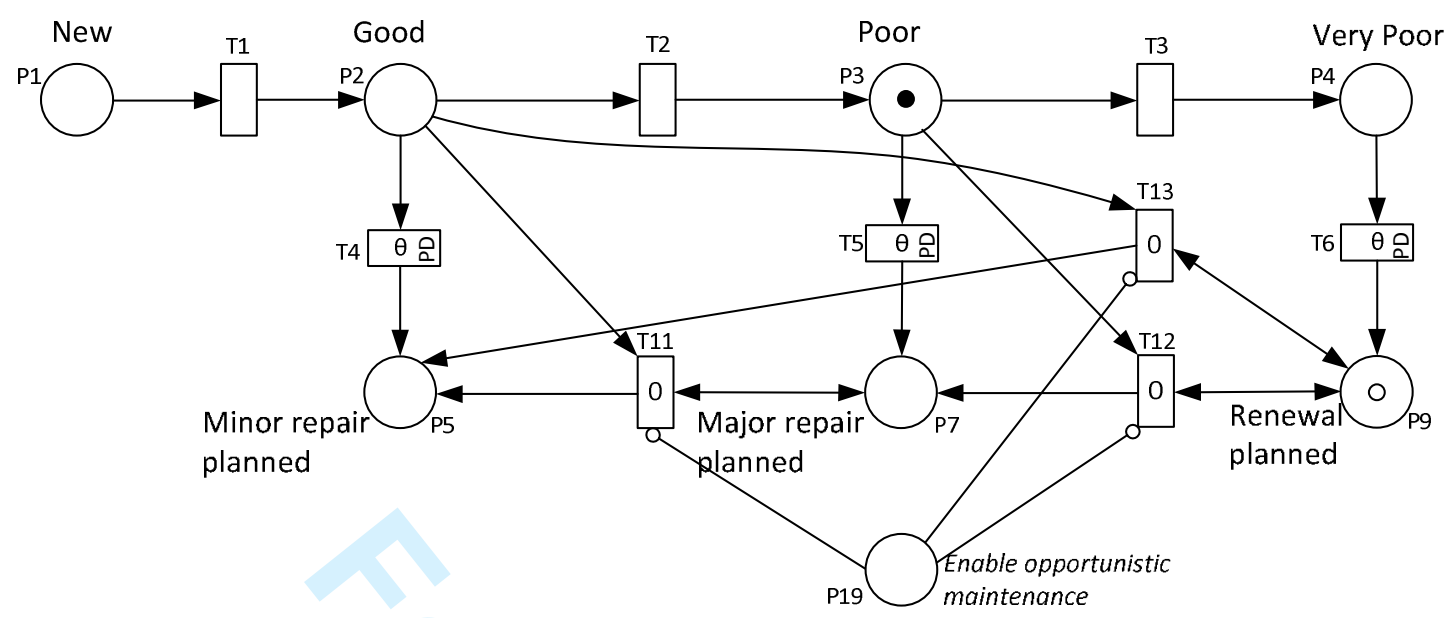

Figure 9: PN module representing opportunistic maintenance

Figure 9 illustrates the PN representation to model opportunistic maintenance. In the model, the black and white tokens represent two separate bridge main girders. The white token is in place P9, this means that one girder is in the very poor condition and is scheduled for replacement. The other girder, represented by the black token, is shown in the poor state (P3). Since work is to be performed on the girder in the very poor condition, an opportunity is presented to carry out a major repair on the girder identified by the black token. This is modelled by introducing transition T12 which, unlike the usual transitions, has firing rules dependent upon different coloured tokens. The black and white tokens enable transition T12 and after the transition fires, the black token is transferred to place P7 where it is scheduled for a major repair. The white token is cleared from place P9 when the transition fires but is then deposited back in place P9 immediately after the firing as indicating by the double ended arc connecting place P9 to transition T12. Similarly, transitions T11 and T13 are added to model the potential for opportunistic minor repair when there is a component undergoes major repair or replacement. Transitions T11, T12 and T13 are conditional transition, with zero time delay. The zero firing time is to ensure that opportunistic maintenance is implemented immediately after the scheduling of an intervention. It is also important to note that, the conditional property also applies to where the different coloured tokens are deposited after firing. A particular example applies for T12 and governs that the white token is deposited in place P9 after firing, not in place P7. In the implementation of opportunistic maintenance in this model it is only considered based on the scheduled activity for similar components. Thus, transitions T11, T12 and T13 are enabled only when the tokens model the same type of component. A broader implementation can be incorporated where opportunistic maintenance on one element can be performed when work is scheduled on other component types should this be required. Figure 9 also incorporates a means to turn on and off this feature. Place P19 connects to transitions T11, T12 and T13 with inhibitor arcs. Tokens can be added to this place to disable the opportunistic maintenance option in the model. When there is no token, then the opportunistic maintenance is implemented whenever possible. 
4.6 Intervention options

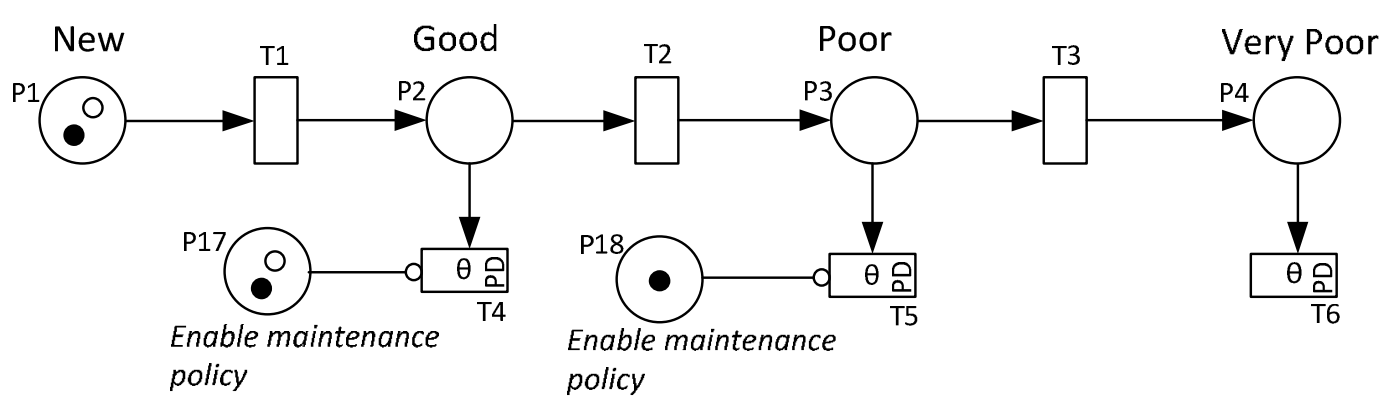

Figure 10: Applying intervention option to individual bridge element in the Petri-net bridge model

As with the opportunistic maintenance, different intervention strategies can also be turned on and off in the model. Consider the PN illustrated in Figure 10. The intervention strategy can be set by placing tokens into places P17 and P18. These places inhibit the transitions T4 and T5 respectively and disable a certain type of repair for a bridge component. In this way the intervention strategy can be applied differently on each bridge component. The black tokens in places P17 and P18 represent the scenario where only renewal is possible for the bridge element modelled by the black token. The white token in place P17 represents the scenario where the component is allowed to deteriorate past the good state and interventions only happen at and beyond the major intervention level. Possible strategies are presented in Table 2. These strategies are applied to individual components. The maintenance strategy for the whole asset is determined by specifying the strategy for all its components.

\begin{tabular}{|c|l|l|}
\hline \multicolumn{1}{|c|}{ Strategy } & \multicolumn{1}{c|}{ PN model representation } \\
\hline $\mathbf{1}$ & $\begin{array}{l}\text { Repair as soon as the component is } \\
\text { identified to be in a state where a repair } \\
\text { is required. }\end{array}$ & Place P17 and P18 are un-marked. \\
\hline $\mathbf{2}$ & $\begin{array}{l}\text { Minor repair is inhibited, only major } \\
\text { repair and replacement is considered. }\end{array}$ & $\begin{array}{l}\text { Place P17 is marked with a token } \\
\text { corresponding to the bridge component } \\
\text { which this strategy is applied to. }\end{array}$ \\
\hline $\mathbf{3}$ & $\begin{array}{l}\text { Major repair is inhibited, only minor } \\
\text { repair and replacement is considered. }\end{array}$ & $\begin{array}{l}\text { Place P18 is marked with a token } \\
\text { corresponding to the bridge component } \\
\text { which this strategy is applied to. }\end{array}$ \\
\hline $\mathbf{4}$ & $\begin{array}{l}\text { Minor and major repair are inhibited, } \\
\text { only replacement is considered. }\end{array}$ & $\begin{array}{l}\text { Place P17 and P18 are marked with a } \\
\text { token corresponding to the bridge } \\
\text { component which this strategy is } \\
\text { applied to. }\end{array}$ \\
\hline
\end{tabular}

Table 2: Possible intervention strategies for a single bridge component.

\subsection{Complete bridge model}

The final bridge model is constructed by connecting the individual PN sections constructed to model each of the aspects described throughout section 4. Performing this process results in the PN structure for an element of the bridge shown in Figure 11. Using the same net, a complete bridge can be modelled by adding more tokens where each token represents a 
unique bridge element. If the component is a metal element with a protective coating, a linked token is added to the coating net.

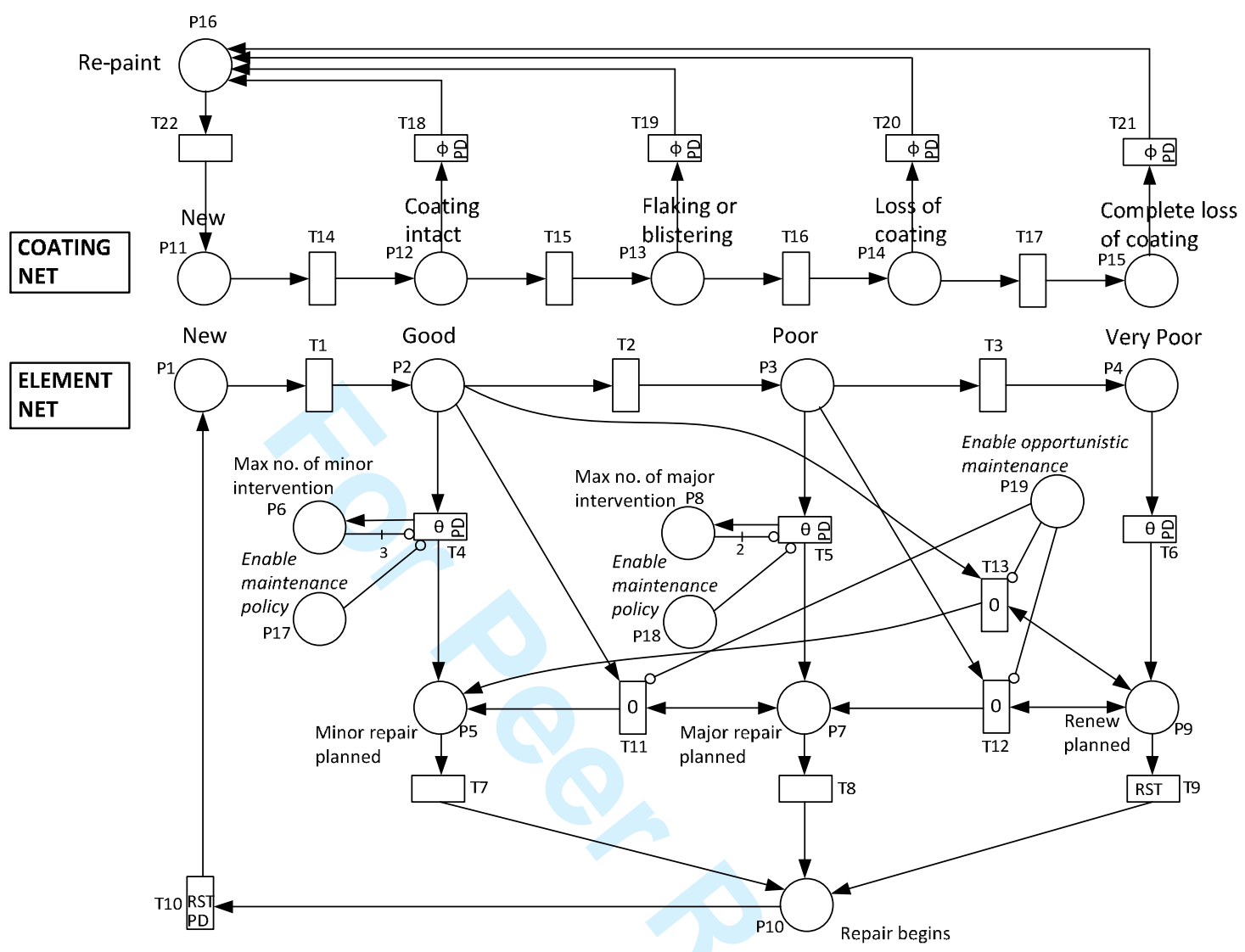

Figure 11: Complete bridge model.

\section{Petri net model analysis, results and discussions}

\subsection{Asset selected}

The bridge asset selected to demontsrate the capabilities of the modelling approach is a typical metal underbridge. The main bridge elements and their initial conditions are described in Table 3:

\begin{tabular}{|l|c|c|}
\hline \multicolumn{1}{|c|}{ Component } & Material & Initial condition \\
\hline Deck (DCK) & Concrete & Good \\
\hline External main girder 1 (MGE 1) & Metal & Good \\
\hline Internal main girder (MGI) & Metal & Poor \\
\hline External main girder 2 (MGE 2) & Metal & Good \\
\hline Bearing 1 (BGL 1) & Metal & Poor \\
\hline Bearing 2 (BGL 2) & Metal & Poor \\
\hline Abutment 1 (ABT 1) & Masonry & As New \\
\hline Abutment 2 (ABT 2) & Masonry & As New \\
\hline
\end{tabular}

Table 3: Asset major components and initial conditions 


\subsection{Model simulation and convergence}

The model is formulated to simulate the dynamic processes which change the states of elements which make up the bridge asset. These changes follow stochastic processes which occur randomly over time. Therefore, model simulation is required and the developed PN bridge model provides the simulation framework. The Monte Carlo simulation [29] procedure was used in which random sampling of the transition times was performed from appropriate distributions. A computer program was written to accommodate the generation and solution of the PN bridge model. The model is then used to simulate the conditions of the bridge and its elements along with the effects of maintenance over a 60 year lifetime period. Figure 12 shows an example of the bridge deck life over a simulated life of 60 years. The graph demonstrates a simulated life of the bridge deck in terms of the time it resides in any condition state before moving to a worse condition (degradation process) or moving to the 'as good as new' condition (repair process). Over the simulated life time, the time that the token resides in each place in the model can be tracked. Carrying out this simulation for a number of times, statistics are then collected to provide a performance indication of each bridge element.

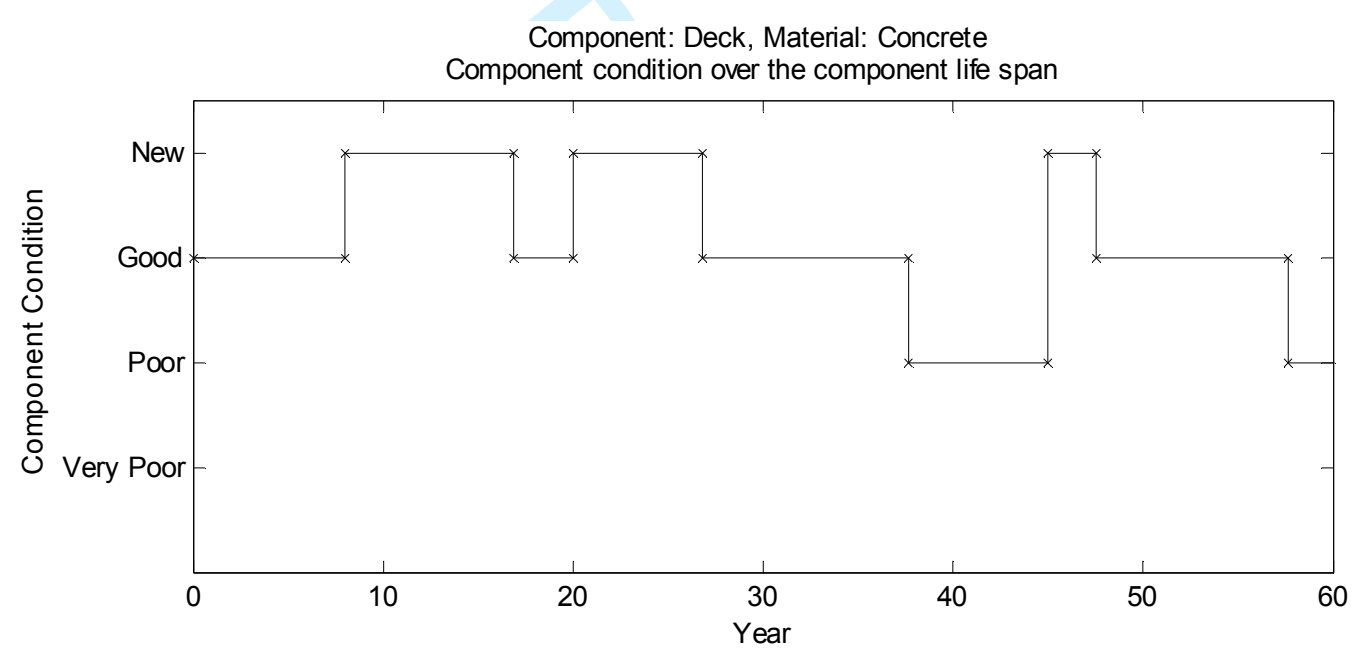

Figure 12: Example of one simulated life time of a bridge deck residing in different condition states

With random sampling of the transition time distributions, the confidence in the performance statistics determined from the model increases with the number of simulations. Running more simulations gives more precise results, but it is also time-consuming. Convergence occurs when running further simulations does not significantly change the model statistics evaluated. By setting a threshold tolerance level it can be established when convergence is achieved. To obtain results from the analysis of this case study example, a 1\% change threshold was used for all of the recorded performance statistics. Convergence was then achieved following around 200 lifetime simulations.

\subsection{Model inputs and parameters}

The model Weibull distribution input parameters used are given in Table 4-Table 7. Table 4 shows the distributions of degradation transition times for the different bridge components. 
These distributions are established from the degradation analysis described in section 2 . For metal components, the deterioration rates are dependent on the condition of the coatings hence the distributions of transition times are different at different coating conditions as shown in Table 5. It can also be seen from the table that the eta value decreases as the as the coating condition worsen, this demonstrates the effect of a slightly faster time of a metal element achieving a poorer condition when the protective paint/coating has degraded. The Weibull distributions for the transition times between each coating condition are given in Table 6.

Table 7 shows the constant, periodical transition times, in years, associated with T4-6 and T18-21. The inspection period is set to every 6 years and maintenance is scheduled every year (T10). The times for transitions T7-9 are 1, 2 and 3 years respectively. These times represent the planning times in each type of maintenance (minor, major repair and renewal). The time for transition T22 is the time it takes to restore the coating condition back to new and was assumed to be one week.

\begin{tabular}{|c|c|c|c|c|c|c|c|c|}
\hline \multicolumn{9}{|c|}{ Stochastic transition time (years) } \\
\hline Component & \multicolumn{2}{|c|}{ Girder } & \multicolumn{2}{c|}{ Decking } & \multicolumn{2}{c|}{ Bearing } & \multicolumn{2}{c|}{ Abutment } \\
\hline Material & \multicolumn{2}{|c|}{ Metal } & \multicolumn{2}{c|}{ Concrete } & \multicolumn{2}{c|}{ Metal } & \multicolumn{2}{c|}{ Masonry } \\
\hline Transition ID & Beta & Eta & Beta & Eta & Beta & Eta & Beta & Eta \\
\hline T1 & 1.71 & 23.39 & 1.08 & 19.09 & 0.84 & 14.94 & 1.00 & 51.94 \\
\hline T2 & 3.42 & 27.10 & 2.95 & 11.00 & 3.27 & 5.00 & 3.47 & 53.00 \\
\hline T3 & 3.78 & 114.20 & 2.49 & 14.30 & 3.53 & 9.50 & 3.25 & 71.6 \\
\hline
\end{tabular}

Table 4: Transition rates for Transition T1, T2 and T3 depending on the type of token (component type and component material)

\begin{tabular}{|c|c|c|c|c|c|c|c|c|c|c|}
\hline $\begin{array}{c}\text { Metal } \\
\text { Element }\end{array}$ & \multicolumn{9}{|c|}{ Stochastic transition time (years) } \\
\hline $\begin{array}{c}\text { Coating } \\
\text { condition }\end{array}$ & \multicolumn{1}{|c|}{ New Coating } & \multicolumn{2}{|c|}{ Coating intact } & \multicolumn{2}{|c|}{$\begin{array}{c}\text { Flaking or } \\
\text { blistering }\end{array}$} & \multicolumn{2}{|l|}{ Loss of coating } & \multicolumn{2}{c|}{$\begin{array}{c}\text { Complete loss of } \\
\text { coating }\end{array}$} \\
\hline Transition ID & Beta & Eta & Beta & Eta & Beta & Eta & Beta & Eta & Beta & Eta \\
\hline T1 & 1.71 & 23.39 & 1.71 & 22.2 & 1.71 & 21.05 & 1.71 & 19.88 & 1.71 & 17.78 \\
\hline T2 & 3.42 & 27.10 & 3.42 & 25.7 & 3.42 & 24.39 & 3.42 & 23.04 & 3.42 & 20.60 \\
\hline T3 & 3.78 & 114.20 & 3.78 & 108 & 3.78 & 102.78 & 3.78 & 97.07 & 3.78 & 86.79 \\
\hline
\end{tabular}

Table 5: Transition rates for Transition T1, T2 and T3 for Metal element depending on the condition of the coating

\begin{tabular}{|c|c|c|}
\hline Metal Coating & \multicolumn{2}{|c|}{ Stochastic transition time (years) } \\
\hline Transition ID & Beta & Eta \\
\hline T14, 15, 16, 17 & 1.0 & 5 \\
\hline
\end{tabular}

Table 6: Transition rates for the coating of metal element (Transition T11 - T14)

\begin{tabular}{|c|c|c|c|c|c|c|c|c|}
\hline Transition ID & T4-6 & T10 & T18-21 & T7 & T8 & T9 & T22 & T11-13 \\
\hline Fix transition time (years) & 6 & 1 & 6 & 1 & 2 & 3 & 0.01 & 0 \\
\hline
\end{tabular}

Table 7: Fixed transition times for periodical transition (T4-6, T10, and T18-T21) and constant delay time transition (T7-9, T22 and T11-13)

\subsection{Element analysis}

In the PN bridge model, a bridge component is represented by a token, by tracking the token, the simulation statistics give the predicted component performance. The bridge deck is used 
to illustarte the information generated by the model at component level. Figure 13 shows the mean time of the bridge deck residing in the different condition states. It resides in the 'as new' state for around 40 years over the 60 years simulation period. The plot also shows that convergence was achieved, in this case after around 120 simulations.

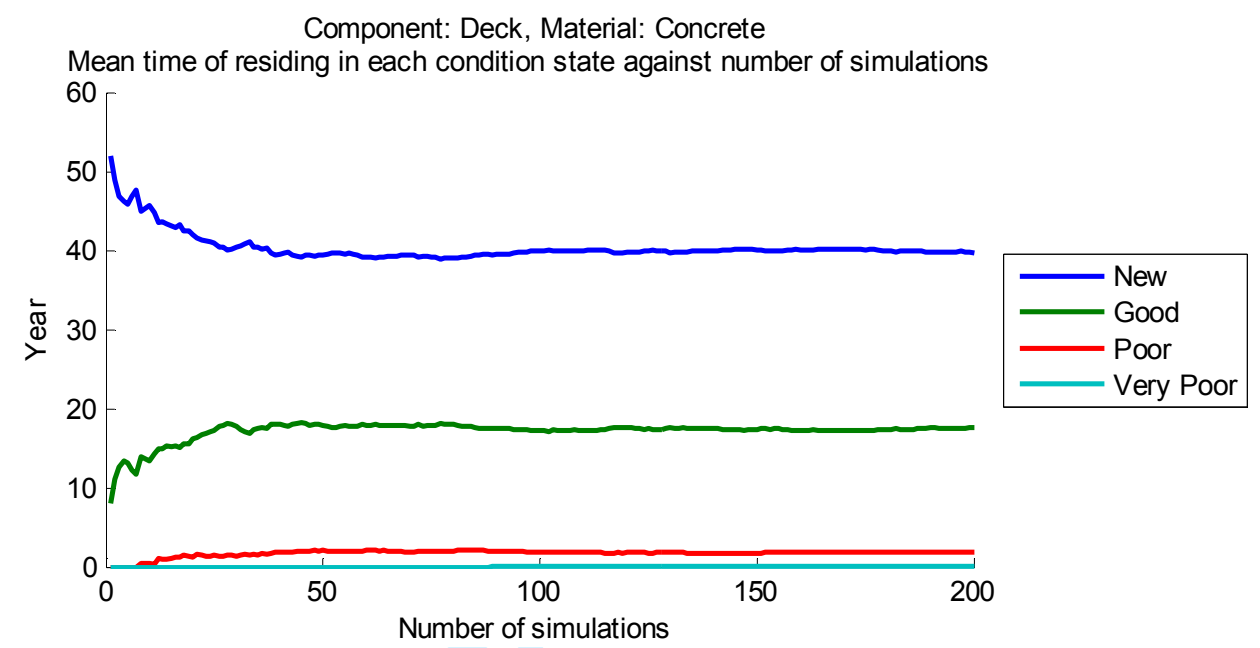

Figure 13: Duration of staying in each condition states against the number of simulation bridge deck - maintenance strategy 1: repair as soon as possible

Figure 14 shows the expected number of each type of intervention to be carried out over the life time of the componet. With the maintenance strategy selected such that repair is carried out as soon as any defect condition is revealed, the expected number minor repairs is the most frequent and is performed around 2-3 times over 60 years period. It is predicted that there is no deck replacement, this agrees with the fact that the expected probabilty of the deck being in a very poor condition is almost zero.

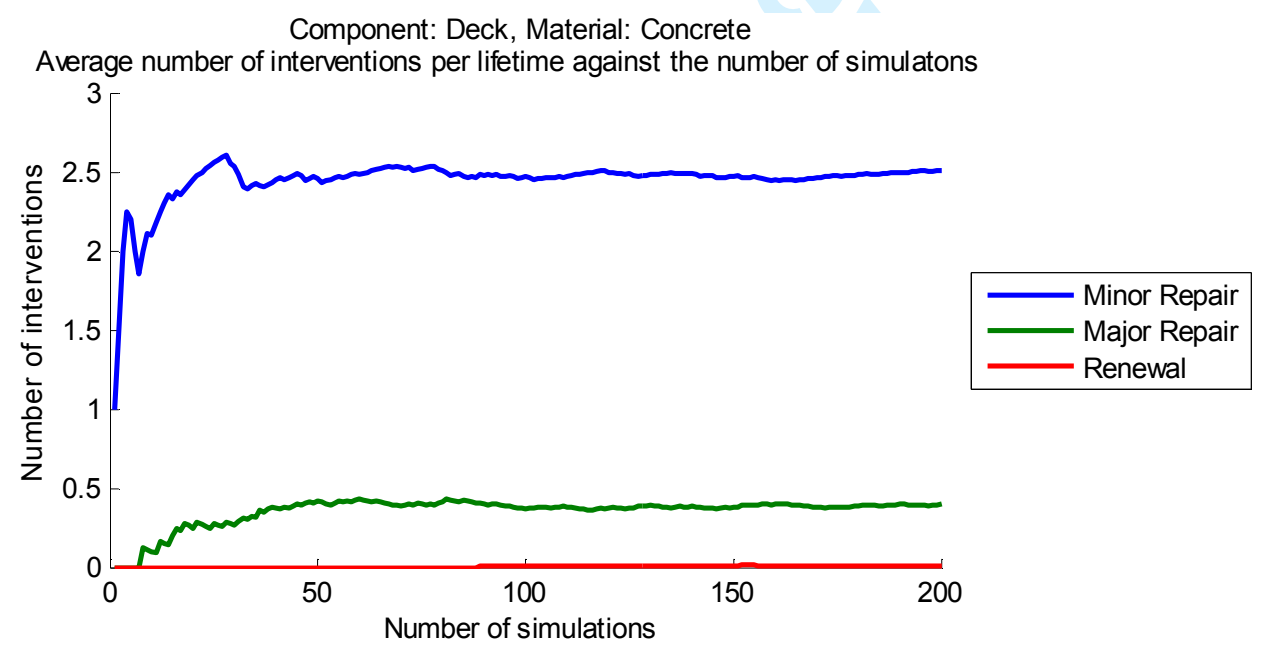

Figure 14: Average number of interventions per lifetime against the number of the simulations - bridge deck - maintenance strategy: repair as soon as possible

Figure 15 shows the bridge deck condition distribution at the end of each year. As the initial condition is good, the probability of the bridge deck being in this condition at the start of the simulation (year 0 ) is 1 . In the immediately proceeding years, this probability decreases 
because the deck starts to deteriorate and the probability of the deck being in the poor condition increases. The first inspection happens at the $6^{\text {th }}$ year, and the condition of the deck is revealed. In those instances in the simulations where it is found in the state where repair is possible, the appropriate repair action is scheduled and carried out to restore the deck condition. The effect is reflected on the plot by the increasing probability of the deck residing in the as new state. Note that the effect does not happen immediately after 6 years because there is a planning time associated with the repair process ( 1 to 3 years depending on the type of repair). Therefore the significnat increases in the probabilty of being in the 'as new' state can be seen to happen around the $7^{\text {th }}$ to $9^{\text {th }}$ year. Carrying on further into the predicted life time, the deterioration process as well as inspection and maintenance process is reflected in the wave nature of the plot.

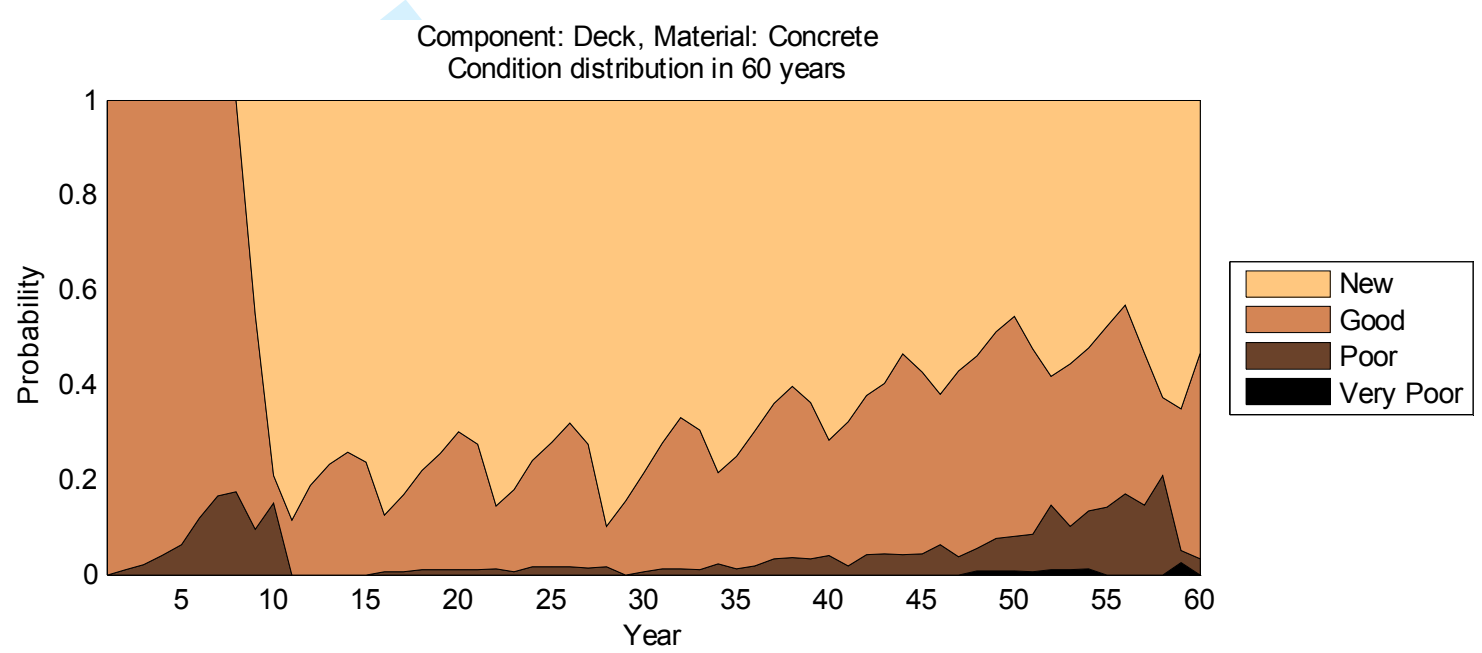

Figure 15: Condition distribution at the end of each year for the bridge deck - maintenance strategy: repair as soon as possible

\subsection{System analysis}

Table 8 and 9 show the summary of the system statistics obtained for all bridge elements when applying maintenance strategy of intervening as soon as any degraded state is discovered. It can be seen that, it is predicted that at least one minor intervention is necessary on all components over their lifetimes. Also, with this maintenance strategy, the average time that the bridge is in the 'as new' condition is roughly around 40 years over the 60 years prediction period. This detailed information allows the investigation of the effects of different specified maintenance strategies in terms of performance and cost.

\begin{tabular}{|c|c|c|c|c|c|c|c|c|c|}
\hline & & DCK & MGE1 & MGI & MGE2 & BGL1 & BGL2 & ABT1 & ABT2 \\
\hline \multirow{4}{*}{ Minor intervention } & Minimum number achieved & 0 & 0 & 0 & 1 & 0 & 0 & 0 & 0 \\
\cline { 2 - 11 } & Maximum number achieved & 4 & 3 & 3 & 3 & 3 & 3 & 3 & 3 \\
\cline { 2 - 11 } & Average & 2.51 & 2.62 & 1.80 & 2.61 & 1.70 & 1.61 & 1.05 & 1.04 \\
\cline { 2 - 10 } & Standard deviation & 0.74 & 0.57 & 0.75 & 0.57 & 1.03 & 1.01 & 0.82 & 0.88 \\
\hline \multirow{4}{*}{ Major intervention } & Minimum number achieved & 0 & 0 & 1 & 0 & 0 & 0 & 0 & 0 \\
\cline { 2 - 10 } & Maximum number achieved & 2 & 1 & 1 & 1 & 2 & 2 & 0 & 0 \\
\cline { 2 - 10 } & Average & 0.40 & 0.03 & 1 & 0.03 & 1.45 & 1.42 & 0 & 0 \\
\cline { 2 - 10 } & Standard deviation & 0.54 & 0.16 & 0 & 0.16 & 0.62 & 0.60 & 0 & 0 \\
\hline Replacement & Minimum number achieved & 0 & 0 & 0 & 0 & 0 & 0 & 0 & 0 \\
\hline
\end{tabular}




\begin{tabular}{|c|c|c|c|c|c|c|c|c|c|} 
Maximum number achieved & 1 & 0 & 0 & 0 & 2 & 1 & 0 & 0 \\
\cline { 2 - 10 } & Average & 0.01 & 0 & 0 & 0 & 0.27 & 0.27 & 0 & 0 \\
\cline { 2 - 10 } & Standard deviation & 0.10 & 0 & 0 & 0 & 0.46 & 0.45 & 0 & 0 \\
\hline
\end{tabular}

Table 8: Statistics on the expected number of interventions on each bridge component maintenance strategy: repair as soon as possible

\begin{tabular}{|c|c|c|c|c|c|c|c|c|c|}
\hline & & DCK & MGE1 & MGI & MGE2 & BGL1 & BGL2 & ABT 1 & ABT 2 \\
\hline \multirow{4}{*}{$\begin{array}{c}\text { As new } \\
\text { condition }\end{array}$} & Min. duration achieved & 14.53 & 17.41 & 26.85 & 20.13 & 13.87 & 10.48 & 41.53 & 31.21 \\
\cline { 2 - 10 } & Max. duration achieved & 52.00 & 52.00 & 51.00 & 52.00 & 51.00 & 51.00 & 60.00 & 60.00 \\
\cline { 2 - 10 } & Average & 39.70 & 42.07 & 41.87 & 42.29 & 37.34 & 37.41 & 54.38 & 54.38 \\
\cline { 2 - 10 } & Standard deviation & 8.14 & 5.98 & 4.60 & 6.12 & 7.72 & 8.27 & 4.57 & 4.91 \\
\hline \multirow{4}{*}{$\begin{array}{c}\text { Good } \\
\text { condition }\end{array}$} & Min. duration achieved & 4.50 & 5.69 & 0 & 8 & 0 & 0 & 0 & 0 \\
\cline { 2 - 10 } & Max. duration achieved & 35.82 & 41.89 & 21.05 & 38.50 & 21.84 & 21.08 & 18.36 & 28.79 \\
\cline { 2 - 10 } & Average & 17.65 & 17.14 & 8.41 & 16.93 & 9.26 & 9.02 & 4.93 & 4.93 \\
\cline { 2 - 10 } & Standard deviation & 6.80 & 5.62 & 4.06 & 5.92 & 5.18 & 5.36 & 4.29 & 4.71 \\
\hline \multirow{4}{*}{$\begin{array}{c}\text { Poor } \\
\text { condition }\end{array}$} & Min. duration achieved & 0 & 0 & 9 & 0 & 2.27 & 2.72 & 0 & 0 \\
\cline { 2 - 10 } & Max. duration achieved & 19.93 & 5.42 & 9.00 & 4.91 & 25.66 & 23.76 & 0 & 0 \\
\cline { 2 - 10 } & Average & 1.89 & 0.09 & 9.00 & 0.08 & 11.47 & 11.45 & 0 & 0 \\
\cline { 2 - 10 } & Standard deviation & 2.82 & 0.62 & 0.00 & 0.55 & 3.93 & 3.90 & 0 & 0 \\
\hline \multirow{3}{*}{$\begin{array}{c}\text { Very poor } \\
\text { condition }\end{array}$} & Min. duration achieved & 0 & 0 & 0 & 0 & 0 & 0 & 0 & 0 \\
\cline { 2 - 10 } & Max. duration achieved & 5.37 & 0 & 0 & 0 & 11.39 & 9.91 & 0 & 0 \\
\cline { 2 - 10 } & Average & 0.04 & 0 & 0 & 0 & 1.23 & 1.41 & 0 & 0 \\
\cline { 2 - 9 } & Standard deviation & 0.40 & 0 & 0 & 0 & 2.34 & 2.47 & 0 & 0 \\
\hline
\end{tabular}

Table 9: Statistics on the duration (years) spending in each condition state of each bridge component - maintenance strategy: repair as soon as possible.

\subsection{Effects of varying intervention strategies on asset condition}

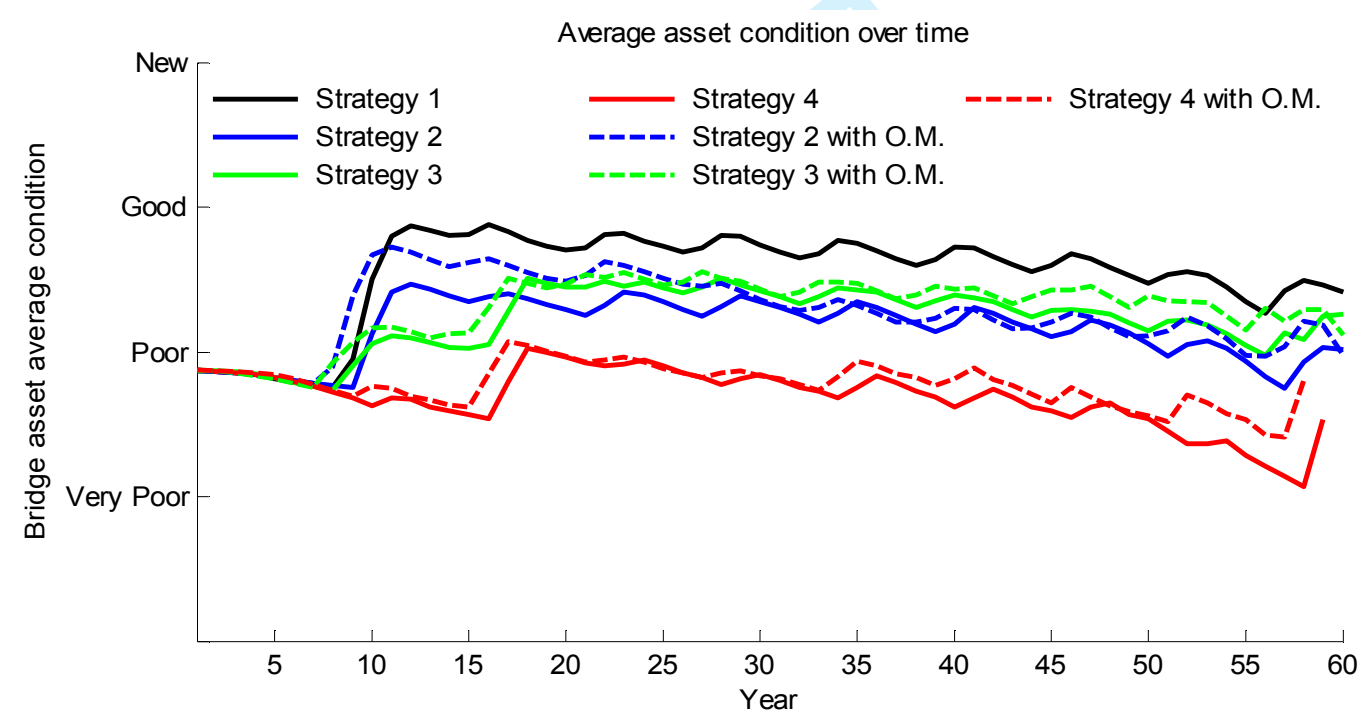

Figure 16: Effects of different intervention strategies with or without opportunistic maintenance (O.M.) on the average asset condition.

The bridge model developed has the capability of accounting for different maintenance strategies. It has been developed so that this can be accommodated simply by depositing tokens in key places at the start of the simulation. This enables the model structure to be retained throughout the analysis process. An assessment has been carried out to investigate the different potential maintenance strategies described. The strategy sets the condition at 
which any element can deteriorate to where restorative maintenance will then be triggered. Figure 16 illustrates the effects of different maintenance strategies in terms of the average asset condition over 60 years prediction period. The four maintenance strategies were simulated are given in Table 2, for strategies 2-4, there are also option to enable opportunistic maintenance. All condition profiles started at the same point as the initial asset average condition is around a poor condition and each component is assumed to be subject to the same strategy. It is obvious for strategies 1, 2 and 4, as the condition that triggers maintenance gets lower at each of the strategy, the predicted asset average condition would also be lower progressively. Since strategy 3 considers replacement and also minor repair, the average asset condition is maintained at a higher condition when compared with strategy 4 and is between predicted average condition for strategies 1 and 2. The plot also illustrates the effects of opportunistic maintenance. Overall, opportunistic maintenance increases the probability of the components being in better conditions, thus maintenance policies with O.M are expected to maintain higher asset average conditions. These are a small subset of the potential asset maintenance strategies since each component of the bridge system can be assigned a different strategy.

\subsection{Expected maintenance costs}

In the system analysis presented in the previous section, it is clear that different strategies are more effective in maintaining a good condition for the bridge system. The more maintenance that is carried out on the structure to achieve this results in higher expenditure. Table 10 shows the expected maintenance cost for all the maintenance strategies considered. This is the total maintenance cost over the 60 years prediction period and is calculated based on the statistics obtained from the analyses reported in the previous sections. It can be seen that strategy 2, which inhibits minor repair, is the most expensive option. This is because, in this case study, the cost of major repair is significantly (about 3 to 5 times) more than the cost of minor repairs. Thus intervention strategies 1 and 3 which allow minor intervention would result in a smaller life cycle cost (LCC). Strategy 4 produced the lowest costs when the components are allowed to deteriorate to a very poor condition. Some of the component's exhibit a long lifetime to reach the very poor state e.g. main girders and abutments. It is expected that these components will not be replaced within the 60 years prediction period, therefore, a low LCC is predicted.

Strategies with opportunistic maintenance enabled have similar predicted LCC compared with their corresponding strategies with no opportunistic repair. Although with strategy 2, a significant saving can be seen by carrying out opportunistic repairs on the external main girders and bearings. The opportunistic maintenance costs are reflected in the cost of minor repairs for these components, which offset the costs of doing more serious and expensive major repairs if the components are allowed to deteriorate further. In this instance the predicted WLCC is actually cheaper by $9.3 \%$ when employing opportunistic maintenance.

Figure 17 and 18 reflect the financial consequences of the intervention strategies. In all strategies, it can be seen that the bearings, initially in a poor condition, contribute a large 
proportion to the total maintenance cost, whereas the expected maintenance costs for the abutments are relatively insignificant.

\begin{tabular}{|c|c|c|c|c|c|c|c|c|c|c|}
\hline Strategy & Intervention type & DCK & MGE1 & MGI1 & MGE2 & BGL1 & BGL2 & ABT1 & ABT2 & Total \\
\hline \multirow{4}{*}{1} & Minor repair & 7455 & 16911 & 11024 & 17106 & 6615 & 7336 & 5423 & 5562 & \\
\hline & Major repair & 3174 & 835 & 23861 & 1432 & 32272 & 32951 & 0 & 0 & \\
\hline & Renewal & 0 & 0 & 0 & 0 & 12000 & 8600 & 0 & 0 & \\
\hline & Total & 10629 & 17746 & 34885 & 18537 & 50887 & 48887 & 5423 & 5562 & 202106 \\
\hline \multirow{4}{*}{2} & Minor repair & 0 & 0 & 0 & 0 & 0 & 0 & 0 & 0 & \\
\hline & Major repair & 14689 & 28037 & 41876 & 28872 & 43935 & 45747 & 4150 & 5321 & \\
\hline & Renewal & 2727 & 0 & 0 & 0 & 26400 & 30000 & 0 & 0 & \\
\hline & Total & 17417 & 28037 & 41876 & 28872 & 70335 & 75747 & 4150 & 5321 & 281303 \\
\hline \multirow{4}{*}{3} & Minor repair & 6631 & 16878 & 98 & 17171 & 5873 & 5938 & 5898 & 6373 & \\
\hline & Major repair & 0 & 0 & 0 & 0 & 0 & 0 & 0 & 0 & \\
\hline & Renewal & 6399 & 0 & 3536 & 0 & 58600 & 60400 & 0 & 0 & \\
\hline & Total & 13030 & 16878 & 3633 & 17171 & 64473 & 66338 & 5898 & 6373 & 203344 \\
\hline \multirow{4}{*}{4} & Minor repair & 0 & 0 & 0 & 0 & 0 & 0 & 0 & 0 & \\
\hline & Major repair & 0 & 0 & 0 & 0 & 0 & 0 & 0 & 0 & \\
\hline & Renewal & 27589 & 416 & 2912 & 1248 & 81200 & 80400 & 0 & 0 & \\
\hline & Total & 27589 & 416 & 2912 & 1248 & 81200 & 80400 & 0 & 0 & 203315 \\
\hline \multirow{4}{*}{\begin{tabular}{l}
\multicolumn{1}{c}{2 with } \\
opportunistic \\
maintenance
\end{tabular}} & Minor repair & 0 & 9789 & 3967 & 9561 & 1397 & 1812 & 587 & 671 & \\
\hline & Major repair & 14364 & 11692 & 33047 & 13601 & 44954 & 45181 & 4150 & 4257 & \\
\hline & Renewal & 3776 & 0 & 0 & 0 & 21200 & 21600 & 0 & 0 & \\
\hline & Total & 18140 & 21480 & 37015 & 23162 & 67551 & 68593 & 4737 & 4927 & 255156 \\
\hline \multirow{4}{*}{$\begin{array}{l}3 \text { with } \\
\text { opportunistic } \\
\text { maintenance }\end{array}$} & Minor repair & 6944 & 17333 & 293 & 17138 & 6572 & 6353 & 5702 & 6066 & \\
\hline & Major repair & 0 & 0 & 119 & 0 & 5888 & 4416 & 0 & 0 & \\
\hline & Renewal & 7763 & 0 & 4575 & 208 & 51000 & 53000 & 0 & 0 & \\
\hline & Total & 14706 & 17333 & 4987 & 17346 & 63460 & 63769 & 5702 & 6066 & 202920 \\
\hline \multirow{4}{*}{$\begin{array}{l}\quad 4 \text { with } \\
\text { opportunistic } \\
\text { maintenance }\end{array}$} & Minor repair & 0 & 0 & 0 & 0 & 589 & 415 & 0 & 0 & \\
\hline & Major repair & 0 & 954 & 358 & 835 & 8832 & 6794 & 0 & 0 & \\
\hline & Renewal & 27799 & 208 & 2288 & 624 & 72000 & 75600 & 0 & 400 & \\
\hline & Total & 27799 & 1162 & 2646 & 1459 & 81422 & 82809 & 0 & 400 & 207247 \\
\hline
\end{tabular}

Table 10: Expected LCC for all bridge components for four maintenance strategies (£)

(a) Strategy 1

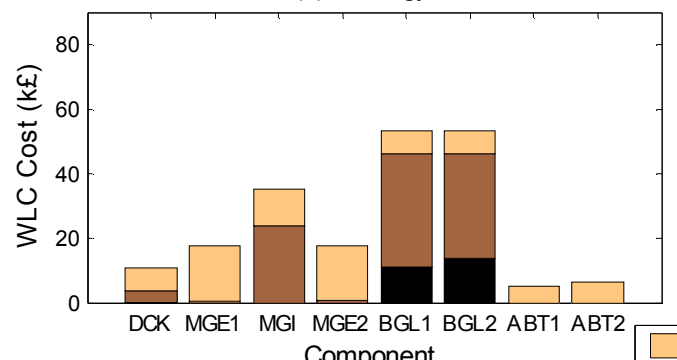

(c) Strategy 3

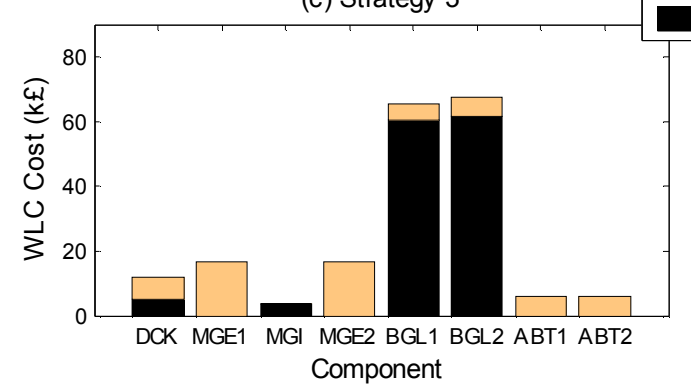

(b) Strategy 2

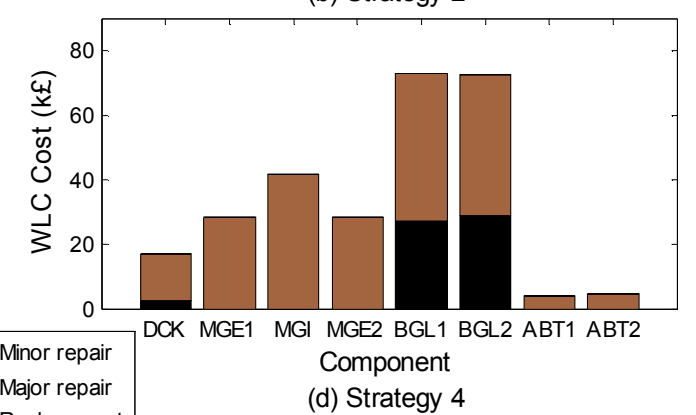

Replacement

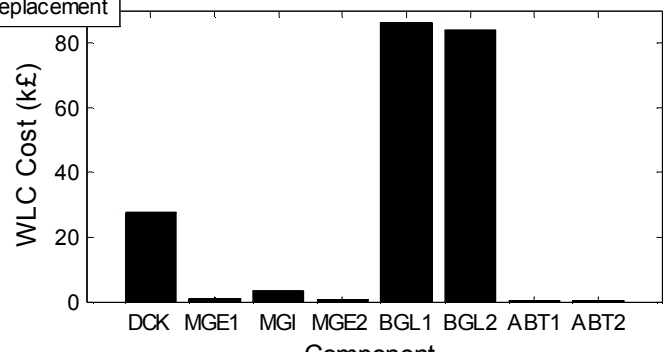

Component

Figure 17: Expected WLCC for each bridge components under four maintenance strategies 
(a) Strategy 2 with opportunistic maintenance

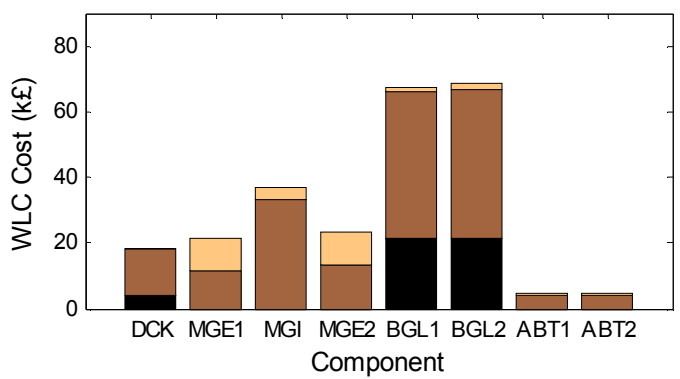

(c) Strategy 4 with opportunistic maintenance

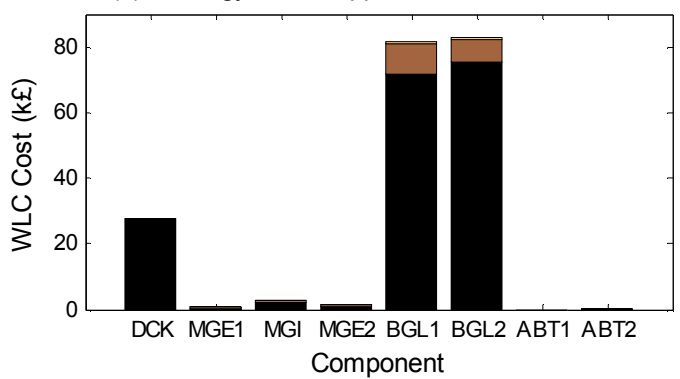

(b) Strategy 3 with opportunistic maintenance

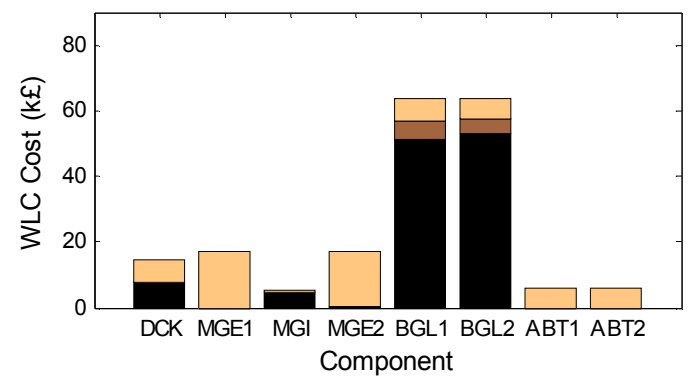

Figure 18: Expected WLCC for each bridge components for strategies 2-4 with opportunistic maintenance

\section{Conclusions}

This paper describes the development of a bridge maintenance modelling methodology based on the Petri Net method. The PN bridge model considers the deterioration, inspection and maintenance of individual components. The deterioration process was derived through the study of historical maintenance records for existing bridge structures of a similar type. The study showed that the Weibull distribution provided a very good fit to the deterioration data and is used to model the times a component takes to reach a specified condition. In this way the modelling overcomes the need to assume a constant deterioration/failure rate which is a feature of many of the earlier modelling approaches. The flexibility of the PN technique allows further features of the structure to be incorporated such as dependent deterioration processes, opportunistic maintenance and a limit on the number of times some maintenance functions can be performed before they become ineffective. The formulation of the model has also facilitated the ability to model different maintenance strategies without the need to change the model structure.

The capabilities of the model have been demonstrated through application to a typical metal underbridge and the range of performance statistics which can be produced to assess the effectiveness and cost of any strategy are presented. From these results it can be seen that the model provides a valuable asset management tool to support the maintenance decision making process.

In particular, the PN bridge model presented has several advantages when compared to the commonly used asset management models. These are: 
- The ability to model non-constant deterioration rates for bridge elements.

- Detail modelling of the individual bridge components along with the consideration of dependent deterioration processes of the coating of metal component on the metal component itself.

- The consideration of the number of effective repetitions of certain repair activities.

- The execution of opportunistic maintenance where the chance to improve the state of one component has presented itself due to the repair being conducted on another.

- The ability to incorporate the planning delays in the maintenance process.

- The modularity properties of the PN model allow more rules to be in incorporated into the model to simulate more complex processes whilst keeping the model size within manageable limits.

\section{Acknowledgement}

John Andrews is the Network Rail Professor of Infrastructure Asset Management. He is also Director of Lloyd's Register Foundation (LRF) ${ }^{1}$ Centre for Risk and Reliability Engineering at the University of Nottingham. Bryant Le and Claudia Fecarotti are conducting a research project funded by Network Rail. They gratefully acknowledge the support of these organisations.

\footnotetext{
${ }^{1}$ Lloyd's Register Foundation supports the advancement of engineering-related education, and funds research and development that enhances safety of life at sea, on land and in the air.
} 


\section{References}

1. Morcous, G., Performance Prediction of Bridge Deck Systems Using Markov Chains. Journal of Performance of Constructed Facilities, 2006. Vol. 20(No. 2): p. 146-155.

2. Jiang, Y. and K.C. Sinha, Bridge service life prediction model using the Markov chain. Transportation Research Record, 1989(1223).

3. Robelin, C.-A. and S.M. Madanat, History-Dependent Bridge Deck Mainenance and Replacement Optimization with Markov Decision Process. Journal of Infrastructure Systems, 2007. 13(3): p. 195-201.

4. Cesare, M.A., et al., Modelling Bridge Deterioration With Markov Chains. Journal of Transportation Engineering, 1991. 118(6): p. 2237.

5. Ortiz-García, J.J., S.B. Costello, and M.S. Snaith, Derivation of transition probability matrices for pavement deterioration modeling. Journal of Transportation Engineering, 2006. 132(2): p. 141-161.

6. Chase, S.B. and L. Gáspár, Modeling the reduction in load capacity of highway bridges with age. Journal of Bridge Engineering, 2000. 5(4): p. 331-336.

7. Fernando, D., B.T. Adey, and N. Lethanh, A model for the evaluation of intervention strategies for bridges affected by manifest and latent deterioration processes. Structure and Infrastructure Engineering, 2015. 11(11): p. 1466-1483.

8. Lethanh, N., B.T. Adey, and D.N. Fernando, Optimal intervention strategies for multiple objects affected by manifest and latent deterioration processes. Structure and Infrastructure Engineering, 2015. 11(3): p. 389-401.

9. $\mathrm{Ng}, \mathrm{S}$. and F. Moses, Prediction of bridge service life using time-dependent reliability analysis. Bridge management, 1996. 3: p. 26-32.

10. Yang, Y.N., H.J. Pam, and M.M. Kumaraswamy, Framework Development of Performance Prediction Models for Concrete Bridges. Journal of Transportation Engineering, 2009. 135(8): p. 545-554.

11. Agrawal, A.K., A. Kawaguchi, and Z. Chen, Deterioration rates of typical bridge elements in New York. Journal of Bridge Engineering, 2010. 15: p. 419-429.

12. Thomas, O. and J. Sobanjo, Comparison of Markov Chain and Semi-Markov Models for Crack Deterioration on Flexible Pavements. Journal of Infrastructure Systems, 2013. 19(2): p. 186-195.

13. Noortwijk, J.M.v. and H.E. Klatter, The use of lifetime distribution in bridge maintenance and replacement modelling. Computers and Structures, 2004. 82: p. 1091-1099.

14. Sobanjo, J., P. Mtenga, and M. Rambo-Roddenberry, Reliability-based modeling of bridge deterioration hazards. Journal of Bridge Engineering, 2010. 15(6): p. 671-683. 
15. Sobanjo, J.O., State transition probabilities in bridge deterioration based on Weibull sojourn times. Structure and Infrastructure Engineering, 2011. 7(10): p. 747-764.

16. Le, B. and J. Andrews, Modelling railway bridge asset management. Proceedings of the Institution of Mechanical Engineers, Part F: Journal of Rail and Rapid Transit, 2013.

17. Office of Rail Regulation, Annual assessment of Network Rail 2006-07. 2007.

18. DeStefano, P.D. and D.A. Grivas, Method for estimating transition probability in bridge deterioration models. Journal of Infrastructure Systems, 1998. 4(2): p. 56-62.

19. Le, B. and J. Andrews. Modelling Railway Bridge Degradation Based on Historical Maintenance Data. in Safety and Reliability. 2015. Taylor \& Francis.

20. Petri, C.A., Fundamentals of a Theory of Asynchronous Information Flow. Proc. of IFIP Congress 62. --- Amsterdam: North Holland Publ. Comp., 1963: p. 386-390.

21. Malhotra, M. and K.S. Trivedi, Dependability modeling using Petri-nets. Reliability, IEEE Transactions on, 1995. 44(3): p. 428-440.

22. Volovoi, V., Modeling of system reliability Petri nets with aging tokens. Reliability Engineering \& System Safety, 2004. 84(2): p. 149-161.

23. Girault, C. and R. Valk, Petri nets for systems engineering: a guide to modeling, verification, and applications. 2002: Springer.

24. Murata, T., Petri nets: Properties, analysis and applications. Proceedings of the IEEE, 1989. 77(4): p. 541-580.

25. Zio, E., Reliability engineering: Old problems and new challenges. Reliability Engineering \& System Safety, 2009. 94(2): p. 125-141.

26. Bause, F. and P.S. Kritzinger, Stochastic Petri Nets. 2002: Springer.

27. Reisig, W. and G. Rozenberg, Lectures on Petri Nets I: Basic Models: Advances in Petri Nets. Vol. 1. 1998: Springer.

28. Andrews, J., A modelling approach to railway track asset management. Proceedings of the Institution of Mechanical Engineers, Part F: Journal of Rail and Rapid Transit, 2013. 227(1): p. 56-73.

29. Rubinstein, R.Y. and D.P. Kroese, Simulation and the Monte Carlo Method, . 2011: Wiley. 